\title{
Simultaneous Determination of Clenbuterol and Salbutamol with a Graphene-Nafion Nanocomposite Modified Electrode
}

\author{
Yanli Zhou ${ }^{1,2, *}$, Huanqing Zhang ${ }^{2}$, Zhu Chang ${ }^{1}$, Baoxian Ye ${ }^{2}$, Maotian Xu ${ }^{1,2, *}$ \\ ${ }^{1}$ Henan Key Laboratory of Biomolecular Recognition and Sensing, College of Chemistry and \\ Chemical Engineering, Shangqiu Normal University, Shangqiu 476000, China \\ ${ }^{2}$ College of Chemistry and Molecular Engineering, Zhengzhou University, Zhengzhou 450001, China \\ *E-mail: zhouyanli@mails.ucas.ac.cn (Y.Zhou), xumaotian@sqnc.edu.cn (M. Xu).
}

doi: $10.20964 / 2016.06 .72$

Received: 14 March 2016 / Accepted: 13 April 2016 / Published: 4 May 2016

\begin{abstract}
A highly sensitive electrochemical sensor for the simultaneous determination of clenbuterol (CLB) and salbutamol (SAL) was fabricated by the modification of graphene-Nafion (GN-Nafion) composite film on a glassy carbon electrode (GCE). Both CLB and SAL caused a pair of redox peaks at the GNNafion/GCE in $0.04 \mathrm{M}$ Britton-Robinson buffer solution. Obvious anodic peak currents for the oxidation of both CLB and SAL were observed under the optimal condition. The difference of oxidation peak potentials for CLB and SAL was about $200 \mathrm{mV}$, which made it possible for simultaneous determination of these compounds using the GN-Nafion/GCE. The detection limit for CLB and SAL was $0.13 \mu \mathrm{M}$ and $0.11 \mu \mathrm{M}(\mathrm{S} / \mathrm{N}=3)$, respectively. The proposed method was applied to simultaneously detect CLB and SAL in pork meat with satisfactory results. Otherwise, the excellent reproducibility, stability and selectivity were obtained at the proposed electrode.
\end{abstract}

Keywords: clenbuterol; salbutamol; graphene; Nafion; nanocomposites; electrochemical sensor

\section{FULL TEXT}

(C) 2016 The Authors. Published by ESG (www.electrochemsci.org). This article is an open access article distributed under the terms and conditions of the Creative Commons Attribution license (http://creativecommons.org/licenses/by/4.0/). 